\title{
Vaginitis y cervicitis en planificación familiar
}

\author{
Dres. Clara Inés Vargas*, Betty Lucy Galindo*, Jorge Enrique Martínez**
}

\begin{abstract}
RESUMEN. Con el fin de determinar los patógenos vaginales y cervicales, y su relación con los métodos de planificación, estudiamos a una población de 300 mujeres que planificaban con anovulatorios o dispositivos intrauterinos y las comparamos con un grupo de 144 mujeres que utilizaban métodos naturales o de barrera para su control natal. Al analizar los resultados observamos que el patógeno más comúnmente aislado de la vagina de las pacientes de los diferentes grupos fue la Gardnerella vaginalis $(38.7 \%)$, que la Candida sp no se presentó con mayor frecuencia en el grupo de mujeres que planificaban con anovulatorios (9\%, control $11 \%$ ), y que la frecuencia de aislamiento de la Chlamydia trachomatis del cervix de las mujeres que planificaban fue en aumento a través del estudio (muestra 1: 4.6\%; muestra 4: 20.9\%), no así el grupo control (muestra 1: $11 \%$; muestra 4: 12\%). (Rev Col Obstet Ginecol 1991; 42(3): 223-226).
\end{abstract}

SUMMARY. With the purpose of establishing the vaginal and cervical pathogens and their relationship with family planning methods, we studied a population of 300 women that used either oral contraceptives (OC) or intrauterine devices (IUD's) and compared them with a control group of 144 women that used natural or barrier methods for birth control.

After analyzing the results from the different groups, the following conclusions were drawn: first, the most commonly isolated pathogen in the patient's vagina was found to be the Gardnerella vaginalis (38.7\%); second, the Candida sp did not present itself more frecuently in the group of women that used OC ( $9 \%$, control group $11 \%)$, a results wich stands in opposition to other researchers conclusions; third, the frecuency of isolation of the Chlamydia trachomatis in the cervix of women that used family planning methods increased throughout the study (sample $1: 4.6 \%$; sample $4: 20.9 \%$ ), a pattern wich was not observed in the control group (sample $1: 11 \%$, sample $4: 12 \%$ ).

\section{Introducción}

La flora normal de la vagina de la mujer cambia durante los diferentes períodos de la vida, encontrándose como germen predominante desde la menarquia hasta la menopausia el Lactobacillus sp el cual al producir el ácido láctico mantiene el $\mathrm{pH}$ de la vagina por debajo de 4, 5, lo cual previene la proliferación de patógenos potenciales (1). También forman parte de esta flora normal otros microorganimos como Bacteroides spp, Peptostreptococcus, Listeria, Ureaplasma urealyticum, Streptococcus Beta hemolitico y Staphylococcus epidermidis $(2,3,4)$. Esta flora varía cuando las mujeres utilizan anticonceptivos orales (AO) o dispositivos intrauterinos (DIU) como método de planificación familiar, aumentándose el número de anaerobios y aerobios, y disminuyendo la concentración de Lactobacillus, situación que ha sido considerada normal en este grupo de mujeres (5), pero que facilita, al alterarse el equilibrio establecido por la flora, que organismos potencialmente patógenos proliferen y produzcan síntomas (1).

Es así como se ha descrito que el desequilibrio producido por los efectos hormonales de los $\mathrm{AO}$ sobre el epitelio vaginal y la flora normal vaginal, hace que las mujeres que planifican con ellos presenten con mayor frecuencia vaginosis bacteriana causada por Gardnerella vaginalis $(5,6)$; durante mucho tiempo también se asoció el uso de $\mathrm{AO}$ con una mayor predisposición a la vaginitis por Candida sp (7),

\footnotetext{
* Bacteriólogas, Grupo Microbiología, Instituto Nacional de Salud, Santafé de Bogotá, D.C.

** M.D. Profamilia, Santafé de Bogotá, D.C. A.A. 80080.
}

sin embargo hoy en día se ha logrado demostrar que esto no es cierto $(1,8)$.

En varios estudios se ha reportado que en el grupo de planificadoras, las usuarias de AO tienen un mayor riesgo de contraer infección por Chlamydia trachomatis debido, posiblemente, a que en estas mujeres se incrementa el epitelio columnar, susceptible a la infección, como también al efecto estimulativo de los esteroides sobre el crecimiento de este microorganismo $(9,10,11,12,13)$, cosa que no sucede en las usuarias del DIU (14).

En algunos de los estudios sobre anticonceptivos orales, se ha visto que estas usuarias tienen un alto riesgo de infección por $N$ gonorrhoeae, aunque no se ha encontrado un mecanismo biológico que explique este riesgo (9).

Las mujeres que planifican con dispositivos intrauterinos tienen también riesgo de sufrir infecciones cérvicovaginales por los cambios en la flora, mencionados anteriormente, y por las guías del dispositivo que permiten la transferencia de bacterias de la cavidad vaginal al interior del útero, el cual colonizan $(4-15)$.

En nuestro medio no encontramos ningún trabajo que demuestre la relación entre planificación y frecuencia de infección por patógenos como $C$ trachomatis, Neisseria gonorrhoeae, $G$ vaginalis, Candida sp y Trichomona vaginalis, por lo tanto quisimos averiguar la frecuencia con que se presentan estas infecciones en nuestra población de planificadoras con anovulatorios o con dispositivos intrauterinos, comparándolas con un grupo de mujeres que planificaban con métodos naturales o definitivos. 
Encontramos que el germen que causa con mayor frecuencia patología en la vagina de las mujeres, es la $G$ vaginalis, sin existir diferencia significativa entre los diferentes grupos de planificadoras, pero sí con el grupo control, y que la frecuencia de infección de la $C$ trachomatis se incrementaba en las mujeres que planificaban y se mantuvo estable en el grupo control.

\section{Material y métodos}

Pacientes. Se estudiaron 444 mujeres sexualmente activas que asistieron a los servicios de planificación de Profamilia entre septiembre de 1984 y diciembre de 1987, cuyas edades oscilaron entre 20 y 25 años, cuyo último parto o aborto ocurrió por lo menos seis meses antes de iniciar el estudio y no presentaban ninguna enfermedad ginecológica ni sistémica.

Las pacientes fueron divididas en cuatro grupos: el primero era el grupo control, integrado por 144 mujeres que utilizaban métodos de planificación naturales como el sintotérmico, de barrera como el condón, o definitivos como la esterilización. El segundo y tercer grupos estuvieron formados por mujeres que iban a planificar con dispositivo intrauterino (159 con TCu 200 B y 108 con Asa de Lippes) el cual se les colocó en el momento de iniciar el estudio. El último grupo estuvo constituido por 33 pacientes que tomaban $\mathrm{AO}$ al comenzar la investigación.

Todas las participantes fueron informadas de las características del estudio y del compromiso de asistir a los controles rutinarios. A las usuarias de dispositivo se les tomó una muestra inicial, un primer control a los 45 días después de haberles colocado la TCu o el Lippes y luego otros dos controles con un intervalo de 3 meses cada uno. A las pacientes del grupo control y las que planificaban con $\mathrm{AO}$ se les tomó la muestra inicial y 3 controles, con una diferencia de 32 meses cada uno.

Toma de muestras. Las muestras fueron tomadas por un médico en un consultorio de Profamilia con asistencia de una auxiliar de enfermería y dos bacteriólogas del Instituto Nacional de Salud. A todas las pacientes se les practicó la citología y estudios microbiológicos cérvicovaginales; para los microbiológicos se tomaron 4 escobillones de vagina, 2 endocervicales y uno rectal (16). Se tomó un muestra de sangre para el VDRL y el FTA-ABS.

Procesamiento. Las muestras fueron procesadas en el Laboratorio de Microbiología del Instituto Nacional de Salud.

El primer escobillón vaginal se colocó en 1 cc de solución salina fisiológica estéril, para observar más tarde la presencia de Trichomonas vaginalis en un examen en fresco (40X). El segundo escobillón se sembró en un caldo especial para cultivar la $T$ vaginalis (17). Con el tercero se inoculó una caja de agar sangre de cordero y una de agar sangre humana bifásico (HB) (18). para el diagnóstico de gérmenes comunes (19) y de $G$ vaginalis $(18,20)$. El cuarto y último escobillón vaginal se sembró en una caja de agar Sabouraud para el diagnóstico de Candida sp (21), con el mismo escobillón se hizo un extendido, se coloreó con Gram para determinar la flora bacteriana y el número de leucocitos por campo microscópico (22).

Uno de los escobillones endocervicales se cultivó sobre Thayer Martin para el diagnóstico de $N$ gonorrhoeae (16). Con un escobillón con base de aluminio se tomó una muestra del canal cervical para el cultivo de la $C$ trachomatis y se colocó en un medio de transporte que consistía en RPMI con suero fetal bovino y antibióticos, se conservó a -70 grados centígrados y luego se sembró sobre células McCoy para observar las inclusiones citoplasmáticas coloreadas con yodo $(23,24)$.

La muestra rectal se procesó de igual manera que la cervical para diagnóstico de $N$ gonorrhoeae (16).

A la muestra de sangre se le hizo el VDRL, y cuando este era positivo, se confirmó el diagnóstico con el FTA$\operatorname{ABS}(25)$.

\section{Resultados}

Un total de 444 mujeres acudieron a la muestra inicial y de ellas sólo 208 asistieron a los tres controles, teniendo una deserción del $53 \%$.

El primer grupo o grupo control inició con 144 mujeres, 75 regresaron al primer control, 56 al segundo control y 41 pacientes al tercer control. En el segundo grupo formado por las que planificaban con $\mathrm{TCu}$, inició con 159 mujeres, de ellas 140 regresaron al primer control, 115 al segundo y 86 tuvieron los tres controles. El grupo de las mujeres que iniciaron planificación con Lippes formaron el tercer grupo constituido por 108 pacientes, 100 acudieron al primer control, 83 al segundo y 66 a los tres controles. El último grupo estuvo formado por las que planificaban con $\mathrm{AO}$, fue el más reducido, inició con 33 pacientes, al primer control regresaron 28 , al segundo 20 y sólo 15 acudieron a los tres controles.

$\mathrm{Al}$ analizar los resultados de la citología cérvicovaginal (CCV) encontramos que en los cuatro grupos la mayoría de las pacientes presentaban una citología en Grado II y sólo dos pacientes de las usuarias de Lippes y una del grupo de AO presentaban displasia severa. Las mujeres con displasia moderada o severa fueron remitidas para el estudio colposcópico y tratamiento ginecológico.

De los patógenos vaginales el que con menor frecuencia se aisló fue la $T$ vaginalis (Tabla 1), no encontrándose diferencia significativa en los diferentes grupos, aunque es de anotar que los porcentajes más altos de aislamiento fueron en los grupos de $\mathrm{AO}$ y control.

El hallazgo de Candida sp (Tabla 2) en la vagina de las usuarias de los diferentes métodos de planificación y el grupo control fue similar en las cuatro muestras, teniendo una frecuencia promedio del $9 \%$.

La tasa de aislamiento de la $G$ vaginalis (Tabla 3 ) fue la más elevada de los microorganismos encontrados en la vagina, presentando una frecuencia alta y constante a lo largo del estudio en los diferentes grupos de planificadoras, mientras que el grupo control tuvo una frecuencia de aislamiento semejante a los otros grupos en la primera muestra, pero esta fue decreciendo en los tres controles siguientes, teniendo una diferencia significativa en el segundo y tercer control (P 0.0087 - P 0.0208) con respecto a los otros grupos.

En el cervix encontramos la $N$ gonorrhoeae en un bajo porcentaje: en el grupo control se encontró en $2 / 144$ (1.4\%) pacientes en la muestra inicial, no volviéndose aislar en todo las otras muestras; en el grupo de las usuarias de $\mathrm{TCu}$ se aisló en $1 / 159(0.6 \%)$ en la muestra inicial y en $1 / 86(1.2 \%)$ en el tercer control; los otros dos grupos (usuarias de Lippes y AO) no presentaron infección por $N$ gonorrhoeae. 
Tabla 1

FRECUENCIA DE AISLAMIENTO DE TRICHOMONAS VAGINALIS EN VAGINA

\begin{tabular}{|c|c|c|c|c|c|c|c|c|}
\hline \multirow{2}{*}{ 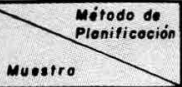 } & \multicolumn{2}{|c|}{$r \in U$} & \multicolumn{2}{|c|}{ LIPPESS } & \multicolumn{2}{|c|}{ AO } & \multicolumn{2}{|c|}{ CONTROL } \\
\hline & $+1 n$ & $\%$ & $+1 n$ & * & $+1 n$ & \% & $+1 n$ & $*$ \\
\hline 1. & $3 / 159$ & $(1,9)$ & $2 / 108$ & $(1,9)$ & $1 / 33$ & $(3,0)$ & $4 / 1144$ & $(2,8)$ \\
\hline 2 & $0 / 0$ & & 11100 & $(1,0)$ & $1 / 28$ & $(3,6)$ & $1 / 75$ & $(1,3)$ \\
\hline 3 & $1 / 115$ & $(0.9)$ & $0 / 0$ & & $1 / 20$ & $(5,0)$ & $2 / 56$ & $(3,6)$ \\
\hline 4 & 010 & & $2 / 66$ & $(3.0)$ & $1 / 15$ & $(6.7)$ & $1 / 41$ & $(2,4)$ \\
\hline
\end{tabular}

Tabla 2

FRECUENCIA DE AISLAMIENTO DE CANDIDA SPEN VAGINA

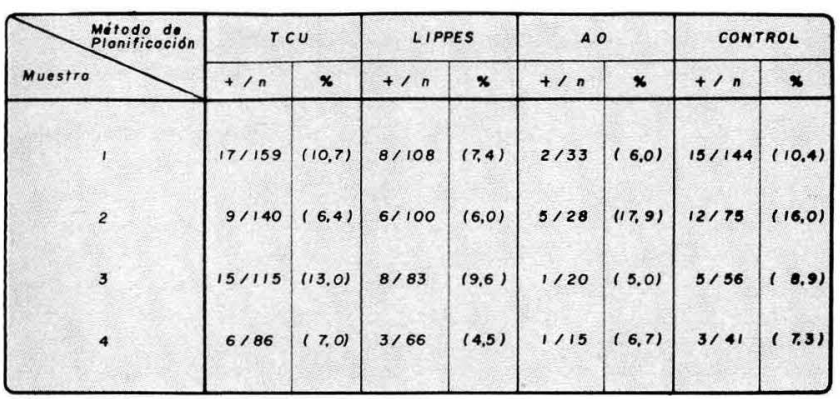

Tabla 3

FRECUENCIA DE AISLAMIENTO DE GARDNERELLA VAGINALIS EN VAGINA

\begin{tabular}{|c|c|c|c|c|c|c|c|c|}
\hline $\begin{array}{l}\text { Mdiodo do } \\
\text { Plonificoción }\end{array}$ & $T C$ & & LIPP & DES & A & & CON & TROLL \\
\hline Muestro & $+1 n$ & $\%$ & $+1 n$ & * & $+1 n$ & * & $+1 n$ & x \\
\hline 1 & $60 / 159$ & $(37.7)$ & $45 / 108$ & $(41,7)$ & $16 / 33$ & $(48,5)$ & $51 / 144$ & $(35,4)$ \\
\hline 2 & $49 / 140$ & $(35,0)$ & $38 / 100$ & $(38,0)$ & $8 / 28$ & $(28,6)$ & $16 / 75$ & $(21,3)$ \\
\hline 3 & $37 / 115$ & $(32,2)$ & $22 / 83$ & $(26,5)$ & $7 / 20$ & $(35,0)$ & $5 / 56$ & $(4,9)$ \\
\hline 4 & 27186 & $(31,4)$ & $20 / 66$ & $(30,3)$ & $3 / 15$ & $(20,0)$ & $3 / 41$ & $(7,3)$ \\
\hline
\end{tabular}

El otro patógeno aislado del cervix fue la $C$ trachomatis; en la primera muestra del grupo control se encontró en una frecuencia mayor $(11 \%)$ con respecto a los otros grupos, y en los tres controles siguientes mantuvo una tasa de aislamiento más o menos constante; en los otros tres grupos (usuarias de $\mathrm{TCu}$, Lippes y $\mathrm{AO}$ ) su aparición fue progresiva terminando el estudio con frecuencias mayores que las pacientes del grupo control (Figura 1).

De los compañeros sexuales de las 136 pacientes, que en total se les diagnosticó infección cervical por $C$ trachomatis, a 70 se les practicó cultivo de secreción uretral, siendo positivos para dicho germen $48(68.6 \%)$.

En la muestra rectal el hallazgo de $N$ gonorrhoeae fue en dos pacientes: una del grupo control en la muestra inicial y la otra del grupo de las usuarias de $\mathrm{TCu}$, en el tercer control; a las dos pacientes también se les había aislado en cervix.

El VDRL fue reactivo en tres pacientes del grupo control, dos en la muestra inicial, y en otra paciente diferente en el primer control; en el grupo de las usuarias de Lippes se encontró en cinco de ellas, dos en la muestra inicial, una en el primer control y dos en segundo control. Todas estas pacientes eran diferentes y su FTA fue positivo.

\section{Discusión}

La $T$ vaginalis tuvo importancia etiológica en la vaginitis de las pacientes de los diferentes grupos, debido a que su hallazgo fue en bajo porcentaje, siendo similar al encontrado por otros investigadores colombianos $(26,27)$.

Con respecto a la vaginitis por Candida sp, Robertson (7) describe que esta infección es más frecuente en mujeres que utilizan AO como método de planificación; en nuestro estudio, este grupo no presentó una mayor frecuencia de aislamiento con respecto al grupo control, ni a los grupos de usuarias de $\mathrm{TCu}$ y Lippes, coincidiendo con lo que citan otros autores $(1,8)$.

El microorganismo que más se aisló como agente etiológico de la vaginosis bacteriana, fue la $G$ vaginalis, encontrándola en porcentajes similares a los de otros estudios (26, 28) donde se considera como el microorganismo patógeno más frecuente de la vagina de las mujeres. También observamos que su presencia en el grupo control fue disminuyendo a lo largo del estudio, lo que no ocurrió en los grupos de las planificadoras, lo que nos sugiere la existencia de una asociación entre la planificación y su prevalencia, sin que haya una tendencia especial hacia alguno de los métodos utilizados.

La infección por $N$ gonorrhoeae sólo fue ocasional en la población estudiada; en el grupo de $\mathrm{AO}$ no hubo pacientes positivas para este micoorganismo, no pudiendo demostrarse la predisposición de estas mujeres a sufrir este tipo de infección como lo describe Louv y colaboradores (9).

En nuestro estudio se observó que la infección cervical por $C$ trachomatis es una de las enfermedades de transmisión

Figura 1

FRECUENCIA DE AISLAMIENTO DE

\section{CHLAMYDIA TRACHOMATIS EN CERVIX RELACIONADA CON METODO DE PLANIFICACION Y NUMERO DE MUESTRAS OBTENIDAS}

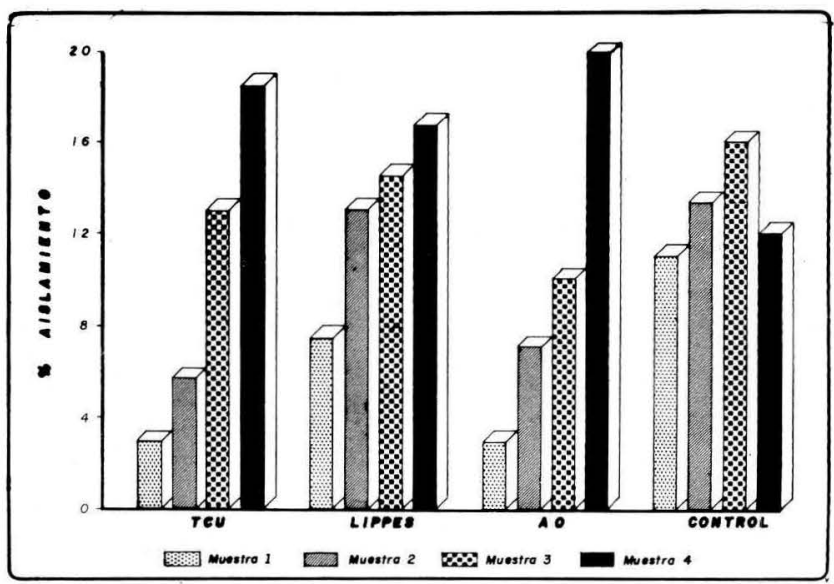


sexual más frecuente, confirmando lo encontrado por nosotros en otro grupo de pacientes (26). Es interesante analizar cómo la infección por $C$ trachomatis se incrementó en las mujeres que planificaban; si comparamos el porcentaje de aislamiento de la muestra inicial de los grupos de $\mathrm{TCu}$, Lippes y $\mathrm{AO}$ con los del tercer control, encontramos un incremento representativo, lo que nos demuestra que las mujeres que planificaban con alguno de estos métodos tienen una mayor tendencia a contraer infecciones cervicales por $C$ trachomatis, no siendo mayor el riesgo en las del grupo de $\mathrm{AO}$.

\section{Agradecimientos}

A Profamilia y al profesor Miguel Trías, quien motivó la realización de esta investigación; al doctor Miguel Guzmán por su asesoría en la primera etapa del trabajo; a los bacteriólogos y biólogos del Grupo de Microbiología Nelly Ordóñez, Danilo Vanegas, Piedad de Forero y Rosa Stella Vargas por su colaboración en el procesamiento de las muestras; al doctor Alfonso Torres y la auxiliar de enfermería Himelda Lemus de Profamilia por su colaboración en la toma de muestras.

\section{BIBLIOGRAFIA}

1. Eschembach D. Infección vaginal. Clínicas Obstet y Ginecolog 1983; 1: $221-240$.

2. Isemberg HD, D'Amato RF. Indigenous and pathogenic microorganisms of humans. In: EH Lennette, A Balows, WJ Hausler, HS Shadomy (eds.), Manual of Clinical Microbiology. Fourth ed. Washington, American Society for Microbiology 1985; 24-35.

3. Normal Microbiol Flora of the Human Body. In: E Sawetz, JL Melmick, EA Adalberg (eds.), Review of Medical Microbiology. Seventeenth edition $1987 ; 314-317$.

4. Sparks RA, Purrier BGA, Watt PJ. The bacteriology of the cervix and uterus. Br J Obstet Gynecol 1977; 84: 701-707.

5. Haukkamaa M, Stranden P, Jousimies H. Bacterial flora of the cervix in women using different methods of contraception. Am J Obstet Gynecol 1986; 154(3): 520-524.

6. Dattani IM, Gerken A, Evans BA. Etiology and management of nos specific vaginitis. Br J Vener Dis 1982; 58: 32-35.

7. Robertson WH. Mycology of vulvovaginitis. Am J Obstet Gynecol 1988; 989-990.

8. Fleury F. Adult vaginitis. Clin Obstet Gynecol 1981; 24: 407-438.

9. Louv WC, Austin H, Perlman J, Alexander J. Oral contraceptive use and the risk of Chlamydial and gonococcal infections. Am J Obstet Gynecol 1989; 160(2): 396-402.

10. Center for Disease Control, Chlamydia trachomatis infection: policy guidelines for prevention and control. MMWR 1985; 34: 53s-74s.

11. Harrison HR, et al. Cervical Chlamydia trachomatis infection in university women: relationship to history, contraception, ectopy, and cervicitis. Am J Obstet Gynecol 1985; 153(3): 244-251.

12. Washington AE, Gove S, et al. Oral contraceptives Chlamydia trachomatis infection, and pelvic inflammatory disease. JAMA 1985 253(15): 2246-2250.

13. Kinghorn GR, Waugh MA. Oral contraceptive use and prevalence of infection with Chlamydia trachomatis in women. Br J Vener Dis 1981; 57: 187-190.

14. Edelman DA. The use of intrauterine contraceptive devices, pelvic inflammatory diseases, and Chlamydia trachomatis infection. Am J Obstet Gynecol 1988; 158(4): 956-959.

15. Sparks RA, Purrier BGA, Watt PJ, Elstein M. Bacteriology colonization of uterine cavity: role of tailed intrauterine contraceptive device. British Med J 1981; 282: 1181-1191.
16. Guzmán M, Vargas CI. Neisseria. En: Microbiología Médica. Manual de procedimientos. Serie de Publicaciones Científicas No. 14. Bogotá, Instituto Nacional de Salud 1988; 127-150.

17. Feinberg JG, Whittington JM. A Culture medium for Trichomonas vaginalis, Donne and Species of Candida. J Clin Pathol 1957; 10: 327-329.

18. Vargas CI, Vanegas D, Heredia R. Gạrdnerella vaginalis. Aspectos Clínicos y de Laboratorio. Serie de Notas e Informes Técnicos No. 18. Bogotá, Instituto Nacional de Salud 1989.

19. Isemberg HD, Washington JA, Balow S, Sonnenwirth AC. Collection, Handling and processing of specimens. En: EH Lennette, A Balows, WJ Hausler, HJ Shadomy (eds.), Manual of Clinical Microbiology. Fourth ed. Washington, American Society for Microbiology $1985 ; 73-98$.

20. Piot P. Gardnerella vaginalis. En: EH Lennette, A Balows, WJ Hausler, HJ Shadomy (eds.), Manual of Clinical Microbiology. Fourth ed. Washington, American Society for Microbiology 1985; 874-876.

21. Cooper BH, Silva-Hunter M. Yeast of medical importance. In: EH Lennette, A Balows, WJ Hausler, HJ Shadomy (eds.), Manual of Clinical Microbiology. Fourth ed. Washington, American Society for Microbiology 1985; 526-541.

22. Spiegel A, Amsel R, Holmes KK. Diagnosis of Bacterial vaginosis by Direct Gram Stain of Vaginal Fluid. J Clin Microbiol 1983; 18(1): 170-177.

23. Bird BR, Forrester ET. Laboratory diagnosts of Chlamydia trachomatis infections. U.S. Department of Healt and Human Services Center for Disease Control. Atlanta. EA 1982.

24. Schachter J. Chlamydia (Psittacosis - Lymphogranuloma venereum - Trachoma group). In: EH Lennette, A Balows, WJ Hausler, HJ Shadomy (eds.), Manual of Clinical Miçrobiology. 4th ed. American Society for Microbiology $1985 ; 856-862$.

25. Guzmán MA. Sífilis, Diagnóstico y Manejo Serológico. Serie de Manuales de Bacteriología Clínica No. 2. Bogotá, Instituto Nacional de Salud 1983.

26. Heredia R, Agudelo CI, Castañeda E. Prevalencia de los agentes etiológicos de la vaginitis y cervicitis en pacientes de la consulta ginecológica general. Acta Médica Colombiana 1990; 15(2): 92-99.

27. Díaz F, Vásquez ME, et al. Vaginitis por Gardnerella vaginalis en un servicio médico universitario. Acta Med Colom 1985; 10(5): 197-203. 